\title{
Treatment With Resveratrol Ameliorates Mitochondrial Dysfunction During the Acute Phase of Status Epilepticus in Immature Rats
}

OPEN ACCESS

Edited by: Ashok Kumar, University of Florida, United States

Reviewed by: Stephanie Wohlgemuth, University of Florida, United States

Sreekanth Puttachary,

Oregon State University,

United States

*Correspondence: Jaroslava Folbergrová jaroslava.folbergrova@fgu.cas.cz

Specialty section: This article was submitted to Neuropharmacology a section of the journal

Frontiers in Neuroscience

Received: 01 December 2020 Accepted: 11 February 2021 Published: 05 March 2021

Citation:

Folbergrová J, Ješina P and Otáhal J (2021) Treatment With Resveratrol Ameliorates Mitochondrial Dysfunction During the Acute Phase of Status Epilepticus in Immature Rats. Front. Neurosci. 15:634378. doi: 10.3389/fnins.2021.634378

\author{
Jaroslava Folbergrová ${ }^{\star}$, Pavel Ješina and Jakub Otáhal \\ Institute of Physiology of the Czech Academy of Sciences, Prague, Czechia
}

The aim of the present study was to elucidate the effect of resveratrol (natural polyphenol) on seizure activity, production of ROS, brain damage and mitochondrial function in the early phase of status epilepticus (SE), induced in immature 12 day-old rats by substances of a different mechanism of action (Li-pilocarpine, DL-homocysteic acid, 4-amino pyridine, and kainate). Seizure activity, production of superoxide anion, brain damage and mitochondrial function were assessed by EEG recordings, hydroethidium method, FluoroJadeB staining and Complex I activity measurement. A marked decrease of complex I activity associated with the acute phase of SE in immature brain was significantly attenuated by resveratrol, given i.p. in two or three doses ( $25 \mathrm{mg} / \mathrm{kg}$ each), 30 min before, 30 or 30 and 60 min after the induction of SE. Increased $\mathrm{O}_{2} \cdot-$ production was completely normalized, brain damage partially attenuated. Since resveratrol did not influence seizure activity itself (latency, intensity, frequency), the mechanism of protection is likely due to its antioxidative properties. The findings have a clinical relevance, suggesting that clinically available substances with antioxidant properties might provide a high benefit as an add-on therapy during the acute phase of SE, influencing also mechanisms involved in the development of epilepsy.

Keywords: reactive oxygen species, immature rats, status epilepticus, superoxide anion production, deficiency of mitochondrial complex I activity, resveratrol, protection

\section{INTRODUCTION}

Existing data clearly indicate that seizures and status epilepticus (SE) are associated with oxidative stress (Patel, 2004; Waldbaum and Patel, 2010; Shin et al., 2011; Folbergrová and Kunz, 2012; Rowley and Patel, 2013; Williams et al., 2015; Folbergrová, 2016). Our recent studies have demonstrated that oxidative stress (demonstrated by the increased production of $\mathrm{O}_{2} \cdot{ }^{-}$in various brain regions) (Folbergrová et al., 2012, 2016) and by the elevation of mitochondrial oxidative damage markers, 3-nitrotyrosine, 4-hydroxynonenal and protein carbonyls) (Folbergrová et al., 2010) and mitochondrial dysfunction (particularly a marked inhibition of respiratory chain complex I activity (Folbergrová et al., 2007, 2010, 2016) occur also in immature brain and may thus be considered a general phenomenon (Folbergrová and Kunz, 2012; Folbergrová, 2013). 
Recent studies suggest that targeting oxidative stress can ameliorate alterations associated with the acute phase of SE and improve also disease outcome (e.g., Pauletti et al., 2017).

Many efforts have been aimed at developing the substances capable of detoxifying reactive oxygen and nitrogen species (ROS and RNS) and their damaging effects (Linseman, 2009; Batinic-Haberle et al., 2010). Synthetic metalloporphyrin catalytic antioxidants (small molecule mimics of SOD and/or catalase) have appeared as a novel neuroprotective agents (Patel and Day, 1999; Reboucas et al., 2008; Sheng et al., 2014). Oxidative stress and neuronal damage associated with status epilepticus in adult animals could be attenuated by some of these compounds (Rong et al., 1999; Liang et al., 2012). We have shown that both superoxide anion formation and the deficiency of complex I activity associated with SE in immature rats could be prevented or substantially attenuated with SOD mimic Mn (III) tetrakis (1-methyl-4-pyridyl) porphyrine pentachloride (MnTMPYP, from Calbiochem), a nitroxide antioxidant and the superoxide dismutase mimic 4-hydroxy2,2,6,6-tetramethylpiperidine-1-oxyl (Tempol, from Sigma) and by a peroxynitrite scavenger and decomposition catalyst 5,10,15,20-tetrakis (4-sulfonatophenyl) porphyrinate Iron (III) (FeTPPS from Calbiochem) (Folbergrová et al., 2007, 2010, 2011, 2016; Folbergrová and Kunz, 2012). In addition, treatment with these antioxidants resulted in a partial amelioration of neuronal degeneration associated with SE in immature rats (Folbergrová et al., 2011, 2016).

It is well-recognized, that acquired epilepsy develops in otherwise healthy brain after the initial "epileptogenic insult" (such as status epilepticus, hypoxic-ischemic insults, infection, trauma, stroke etc.). It triggers vast cascade of multilevel processes which in some individuals finally result in occurrence of spontaneous recurrent seizures, i.e., epilepsy. Epileptogenic insult, epileptogenesis and epilepsy most likely represent independent pharmacological targets. Recently we have observed protective effect of a natural polyphenolic compound present in red wine, resveratrol (RSV) (3,5,4'-tri-hydroxy-trans-stilbene), during epileptogenesis, i.e., long period, up to 4 weeks, following the epileptogenic insult, namely Li-Pilocarpine status epilepticus in immature rats (Folbergrová et al., 2018).

The aim of the present study was to discover effect of resveratrol on seizure activity, production of ROS, neuronal damage and mitochondrial function in the early phase of epileptogenic insult itself, i.e., status epilepticus induced in immature rats. For induction of status epilepticus we have utilized 4 substances of a different mechanism of action, namely DLhomocysteic acid, 4-aminopyridine, Li-pilocarpine and kainate, offering the possibility for general conclusions on resveratrol effect during epileptogenic insult.

\section{MATERIALS AND METHODS}

\section{Animals}

Immature 12-day-old male Wistar rats were used for these experiments. Twelve-day-old rats were chosen because of the level of brain maturation which is comparable to the early postnatal period in human infants (Dobbing, 1970). The rat pups were removed from their dams $1 \mathrm{~h}$ before the experiment. They were kept in plastic observation chambers on an electrically heated pad at $34^{\circ} \mathrm{C}$ (i.e., the temperature of the nest), with the exception of surgery. The protocol of experiments was approved by the Animal Care and Use Committee of the Institute of Physiology, Academy of Sciences of the Czech Republic, in agreement with Animal Protection Law of the Czech Republic, which is fully compatible with the guidelines of the European Community Council directives 86/609/EEC. The Institute possesses The Statement of Compliance with Standards of Humane Care and Use of Laboratory Animals \#A5228-01 from $\mathrm{NIH}$. All efforts were made to minimize animal suffering and to reduce the number of animals used.

\section{Surgery}

The animals were anesthetized with isoflurane and fixed in a stereotaxic apparatus, modified for rat pups (Folbergrová et al., 2000). For DL-HCA and 4-AP application, bilateral stainless steel guide cannulae (26-gauge, $4 \mathrm{~mm}$ in length, Plastics One, Germany) were stereotaxically implanted $1 \mathrm{~mm}$ above the lateral ventricles (AP:0.7 mm caudal from the bregma; $\mathrm{L}: \pm 1.5 \mathrm{~mm}$; $3.3 \mathrm{~mm}$ from the skull surface). Cannulae were fixed to the skull with dental acrylic. After the surgery animals were returned to their mothers in home cages to recover.

\section{EEG Recordings and Analysis}

After surgery, animals recovered $2 \mathrm{~h}$ and then were connected to the EEG system (Pentusa, TDT, United States) and continual EEG were recorded on $1 \mathrm{kHz}$ and stored for offline analysis. The EEG recording covered baseline $(20 \mathrm{~min})$ and whole period of SE development up to $90 \mathrm{~min}$ duration. Offline analyses were performed in Spike2 (CED, United Kingdom) and Matlab (Mathworks, United States) software. Epileptic spikes were detected as suprathreshold events and quantified in $60 \mathrm{~s}$ long bins.

\section{Seizure Induction}

DL-HCA (from Aldrich, Germany) and 4-AP (SigmaAldrich) were dissolved in sterile saline and the $\mathrm{pH}$ adjusted to $\sim 7.0$, only freshly prepared solutions were used. Bilateral i.c.v. infusions of DL-HCA (600 nmol/side), 4-AP (100 nmol/side) or saline were made in a volume of $0.5 \mu \mathrm{l}$ at a rate of $0.17 \mu \mathrm{l} / \mathrm{min}$ using a SP200i infusion pump (WPI, United States) through stainless steel internal cannulae (33 gauge, $5 \mathrm{~mm}$ in length, Plastics One, Germany), each connected by a polyethylene tube to a $10 \mu \mathrm{l}$ Hamilton syringe. To induce Li-Pilo SE, LiCl (SigmaAldrich) was dissolved in redistilled water and administered i.p. to PD11 immature rats $(127 \mathrm{mg} / \mathrm{kg})$. After $24 \mathrm{~h}$, pilocarpine (SigmaAldrich), dissolved in redistilled water was given i.p. (35 $\mathrm{mg} / \mathrm{kg}$ ) to lithium pretreated pups. To induce kainate SE, KA (Tocris Bioscience, Bristol, United Kingdom) was dissolved in saline and given i.p. (6 mg/kg). Control animals received corresponding volumes of the appropriate vehicles.

\section{The Effect of Resveratrol}

For assessing a potential protective effect of a natural polyphenolic compound resveratrol (RSV), RSV (from Sigma 
Co.) was dissolved in DMSO and then diluted with PBS (final concentration of DMSO 5\%). Only freshly prepared solutions, kept in dark, were used for applications. Resveratrol was given i.p. in two or three doses ( $25 \mathrm{mg} / \mathrm{kg}$ each), $30 \mathrm{~min}$ before, 30 or 30 and $60 \mathrm{~min}$ after induction of SE. The schema of experimental design of the current study can be seen in Figure 1.

\section{Superoxide Anion Determination}

Production of superoxide anion $\left(\mathrm{O}_{2}{ }^{-}\right)$in different brain regions in situ was determined using hydroethidium (Het) method (Bindokas et al., 1996), adopted for immature rats, as described in detail in our previous work (Folbergrová et al., 2012). Het was given by i.p. injection immediately before infusion of DLHCA or 4 -AP and $\sim 15$ min after i.p. administration of LiPilo or KA (final concentration $10 \mathrm{mg} / \mathrm{kg}$ ). Sixty minutes after the application of Het, rat pups were deeply anesthetized with $20 \%(\mathrm{w} / \mathrm{v})$ urethane and transcardially perfused with $0.01 \mathrm{M}$

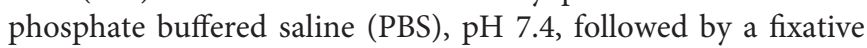
solution $[4 \%(\mathrm{w} / \mathrm{v})]$ paraformaldehyde in $0.1 \mathrm{M}$ phosphate buffer, $\mathrm{pH}$ 7.4. The brains were removed from the skull, postfixed for $3 \mathrm{~h}$ at $4^{\circ} \mathrm{C}$ in the same fixative, then cryoprotected in sucrose of increasing concentrations $(10,20$, and $30 \%(\mathrm{w} / \mathrm{v})$, respectively) in $0.1 \mathrm{M}$ phosphate buffer, $\mathrm{pH} 7.4$ and finally frozen in dry ice. Coronal sections $(50 \mu \mathrm{m})$ were cut through the brain in a cryostat and mounted onto the gelatinated slides. All procedures were performed under the reduced light.

The level of the oxidized products of Het was assessed microscopically by detection of their fluorescence (>600 nm). Pictures of the selected regions of interest (hippocampal fields CA1, CA3, and DG, primary somatosensory cortex and dorsal thalamus) of the same size and orientation, were captured (AP -3.5 to -4.0 according to Paxinos and Watson (Paxinos and Watson, 1998), with cooled camera mounted onto upright microscope (10 x magnification lens). Camera settings remained unchanged throughout the evaluation of the current set of tissue sections of animals from one experimental day, treated with the same solution of Het. The group comprised always at least three saline-treated controls, three animals with convulsant drug alone and three with convulsant drug plus resveratrol. Fluorescence signal (represented as integral intensity of the given region) was normalized by values of the control animals of the current set. Results are expressed as percentage of salinetreated animals.

\section{Brain Damage Analysis}

Brain damage was evaluated in Li-Pilo model of SE. At $24 \mathrm{~h}$ following SE, rat pups from Li-Pilocarpine $(n=9)$ and LiPilocarpine + resveratrol $(n=8)$ groups were subjected to fixation procedure (see its detailed description in the section "Superoxide Anion Determination"). Coronal $50 \mu \mathrm{m}$ thin slices were cut and stained with Fluoro-Jade B (Histochem, United States) as previously used and described in details by our group (Folbergrová et al., 2012, 2016). To assess neurodegeneration and potential protective effect of resveratrol, we have performed semiquantitative grading (using a score) of number of Fluoro-Jade B positive cell in regions of interest (ROI), spatially corresponding to regions evaluated by ethidium method. Neurodegeneration was assessed in hippocampal regions CA1, CA3 and dentate gyrus (DG), sensorimotor cortex (Cx), and mediodorsal thalamic nuclei (Thal). Position of ROIs, selected consistently through all animals, are illustrated in Figure 4B. A semiquantitative scale was used to assess the brain damage; score 0: <7 neurons, score 1: 7-15 neurons, score 2: 16-25 neurons, score 3: 26-40 neurons, score 4: $>40$ neurons.

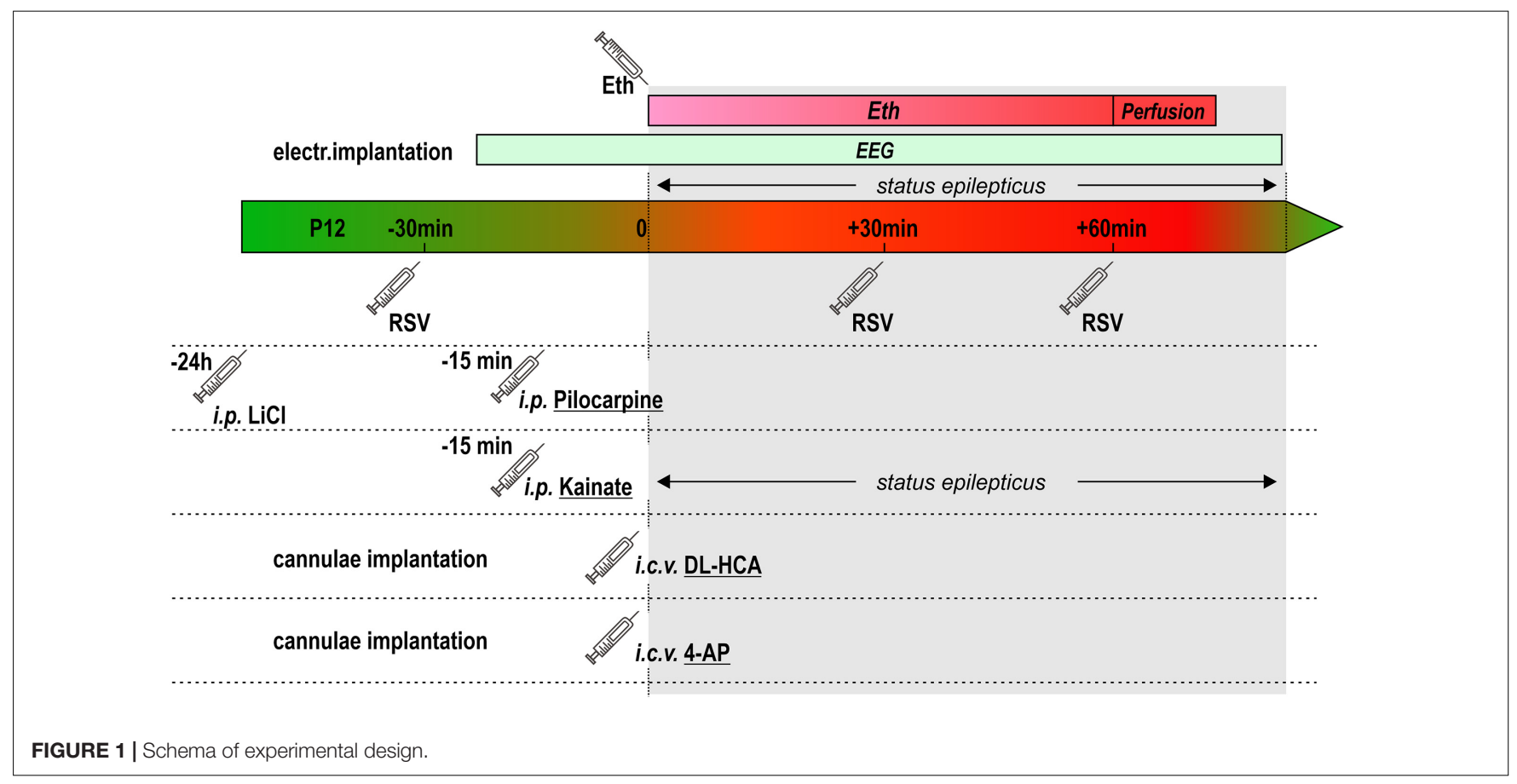




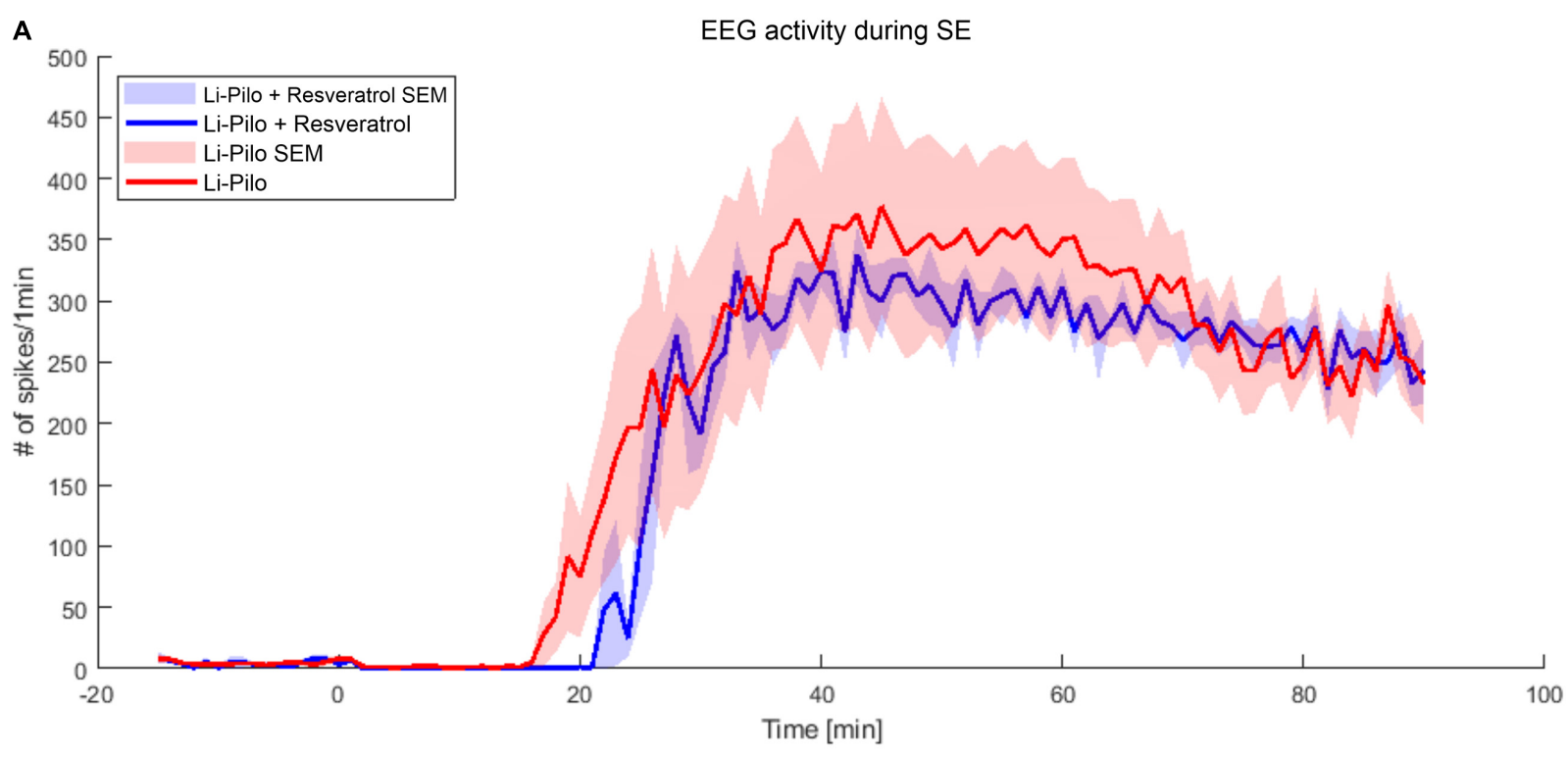

B

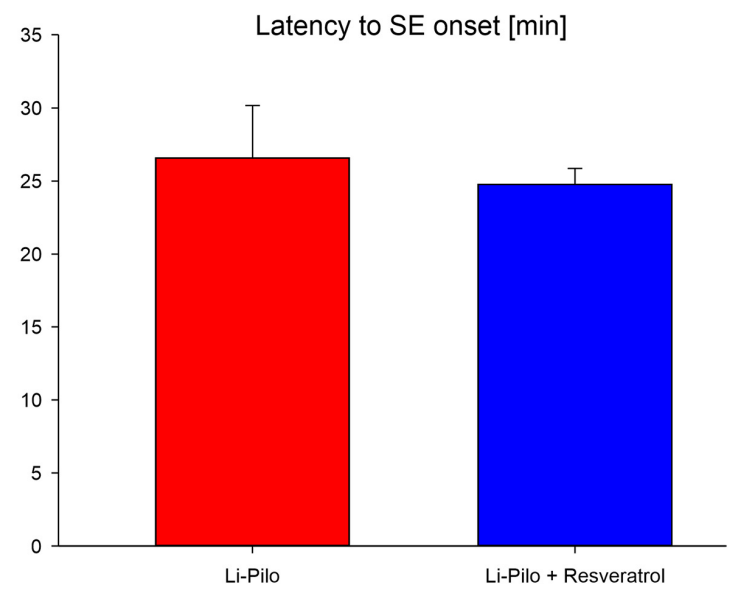

C

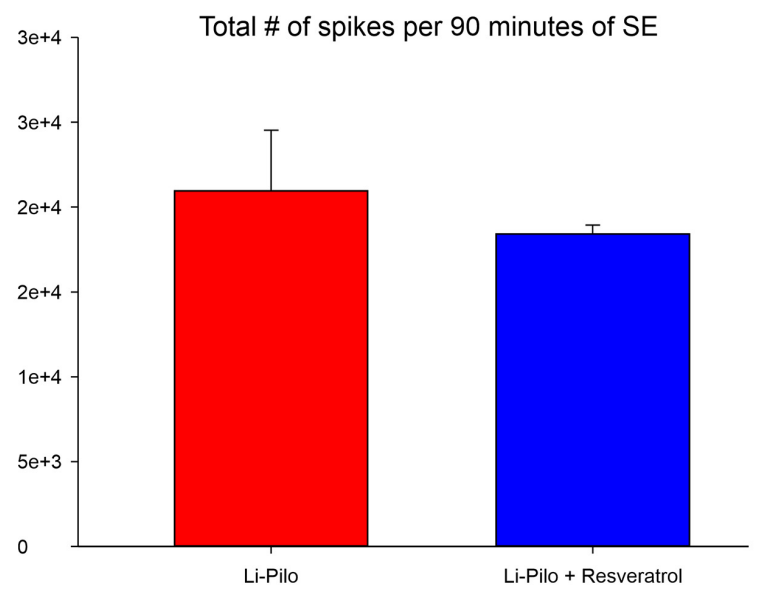

FIGURE 2 | (A) EEG activity during Li-Pilo status epilepticus has been determined as number of epileptic spikes in $60 \mathrm{~s}$ bins. Time zero is the time of pilocarpine application. Red line, convulsant agent alone; Blue line, convulsant agent plus resveratrol. (B) Latency to the first electrographic seizure does not differ after resveratrol treatment $(P=0.753)$. Red column, convulsant agent alone; Blue column, convulsant agent plus resveratrol. (C) Total number of detected epileptic spikes during 90 min of SE duration was not influenced by resveratrol suggesting no anticonvulsant effect $(P=0.668)$. Red column, convulsant agent alone; Blue column, convulsant agent plus resveratrol.

\section{Isolation of Mitochondria}

Mitochondrial fractions were isolated according to Liang et al. (Liang et al., 2000), as described in detail in our previous works (Folbergrová et al., 2007, 2016). All procedures were performed at $4^{\circ} \mathrm{C}$. Cerebral cortices (weighing $\sim 250 \mathrm{mg}$ ) were used for each mitochondrial preparation. $10 \%(\mathrm{w} / \mathrm{v})$ homogenates in ice-cold isolation buffer (70 mM sucrose, $210 \mathrm{mM}$ mannitol, $5 \mathrm{mM}$ Tris$\mathrm{HCl}, 1 \mathrm{~mm}$ EDTA, pH 7.4) were prepared with Elvehjem-Potter type glass-Teflon homogenizers manually by twenty slow upand-down strokes. Homogenates were centrifuged at $600 \times \mathrm{g}$ for $5 \mathrm{~min}$ at $4^{\circ} \mathrm{C}$, the postnuclear supernatant was centrifuged at $17,000 \times \mathrm{g}$ for $10 \mathrm{~min}$ at $4^{\circ} \mathrm{C}$. Mitochondrial pellet was resuspended with $100 \mu 150 \mathrm{mM}$ Tris- $\mathrm{HCl}(\mathrm{pH}$ 7.4). Fresh isolated mitochondria were used for protein determination. Aliquots of mitochondria frozen in liquid nitrogen and stored at $-70^{\circ} \mathrm{C}$ were used for determinations of complex I and citrate synthase activities (performed within 1 week).

\section{Enzyme Assays}

Activities of mitochondrial respiratory chain complex I and citrate synthase were measured at $30^{\circ} \mathrm{C}$ in a total reaction volume of $1 \mathrm{ml}$ using Shimadzu 1601 spectrophotometer. Duplicate determinations were carried out with each mitochondrial sample.

\section{Complex I}

NADH-ubiquinone oxidoreductase (EC 1.6.5.3) activity was determined as the rotenone-sensitive rate of NADH oxidation at $340 \mathrm{~nm}$. The reaction mixture contained: $25 \mathrm{mM}$ potassium 

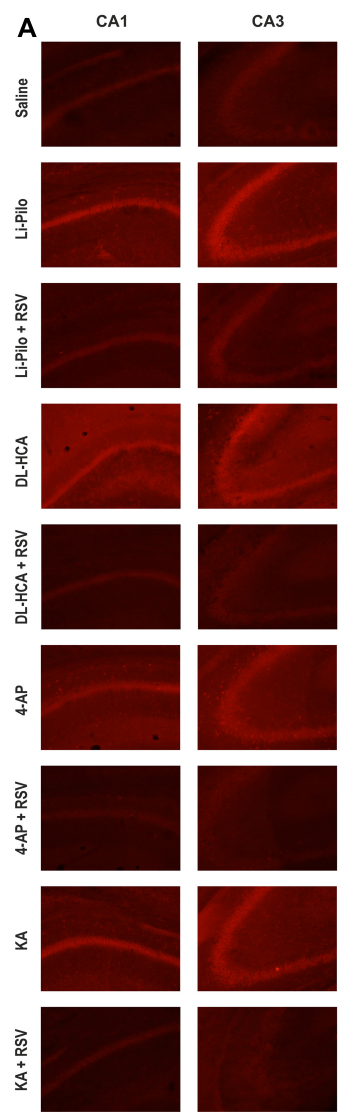
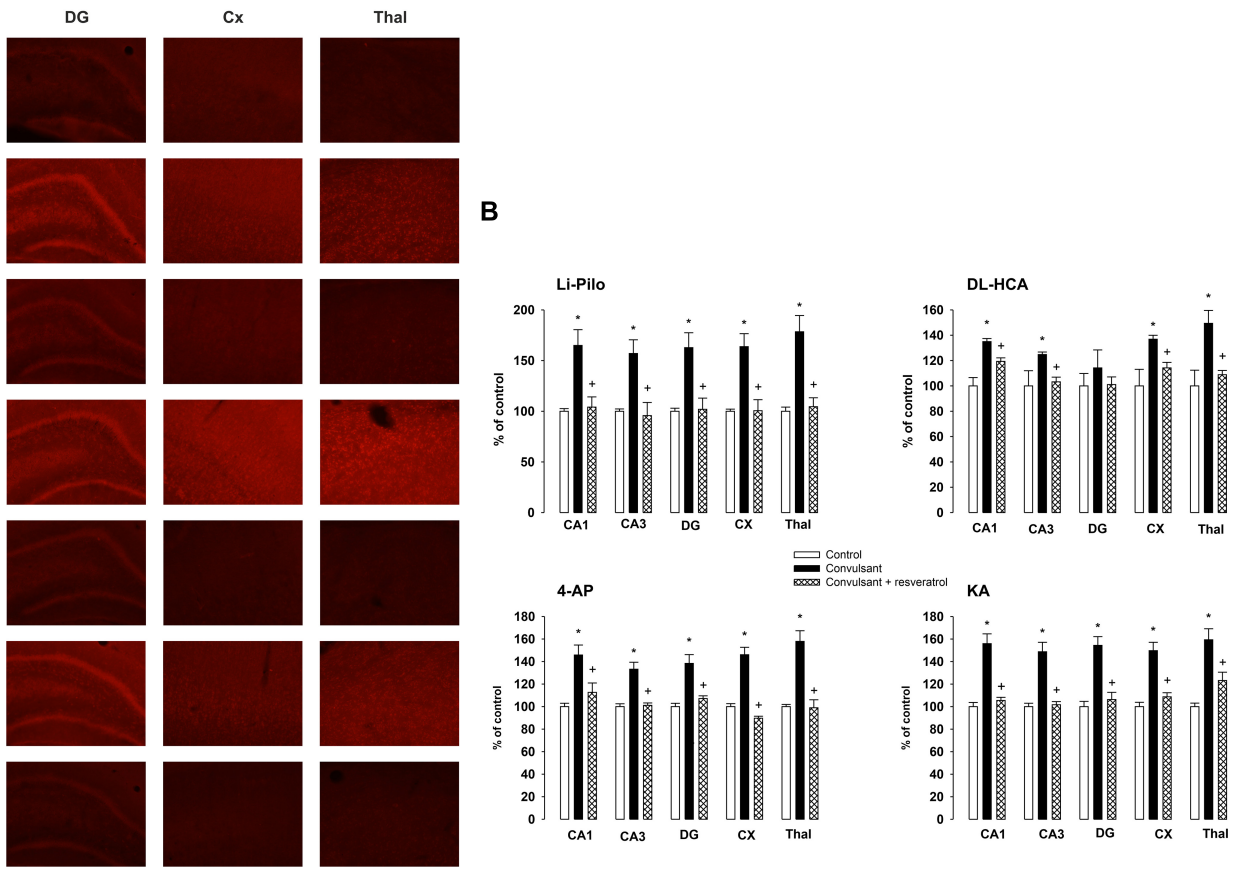

Control
Convulsant
Convulsant

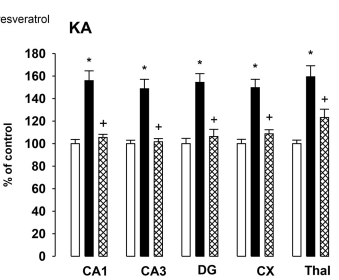

FIGURE 3 | (A) Fluorescence of the oxidized products of hydroethidium (reflecting superoxide anion production), assessed microscopically by fluorescence (> $600 \mathrm{~nm}$ ), in various brain structures following 60 min lasting SE induced by Li-Pilocarpine (Li-Pilo), homocysteic acid (DL-HCA), 4-aminopyridine (4-AP) or kainic acid (KA). Upper image for each model, convulsant agent alone; lower image for each model, convulsant agent plus resveratrol. (B) Effect of resveratrol on superoxide anion formation at 60 min following the onset of SE, induced in immature rats by Li-Pilo, DL-HCA, 4-AP, or KA. White columns, saline-treated controls; black columns, convulsant agent alone; cross-hatched columns, convulsant agent plus resveratrol. Results are expressed in percent, compared to $100 \%$ in the control animals. Mean values for $5-6$ animals \pm SEM. ${ }^{*} P<0.05$ as compared with saline; ${ }^{\dagger} P<0.05$ as compared with convulsant agent alone. CA1 and CA3, hippocampal fields; DG, dentate gyrus; CX, cerebral cortex; Thal, dorsal thalamus.

phosphate ( $\mathrm{pH} 7.2), 10 \mathrm{mM} \mathrm{MgCl}_{2}, 1 \mathrm{mM} \mathrm{KCN}, 0.25 \%$ fatty acid-free bovine serum albumin (BSA), $0.1 \mathrm{mM} \mathrm{NADH}$ and approximately $50 \mu \mathrm{g}$ of mitochondrial protein. The reaction was initiated by the addition of CoQ10 (decylubiquinone, final concentration $50 \mu \mathrm{M}$ ). After $2 \mathrm{~min}, 2 \mu \mathrm{l}$ of rotenone were added (final concentration $5 \mu \mathrm{M}$ ) and the inhibited rate was followed for further 2 min (Folbergrová et al., 2007, 2016).

\section{Citrate Synthase}

Citrate synthase (EC 4.1.3.7) activity was determined as the rate of color change of 5,5'-dithiobis-(2-nitrobenzoic) acid (DTNB) at $412 \mathrm{~nm}$. The reaction mixture contained $100 \mathrm{mM}$ Tris- $\mathrm{HCl}(\mathrm{pH}$ 8.1), 0.2 mM DTNB, 0.1\% Triton X-100, 0.1 mM acetyl-CoA and $\sim 20 \mu \mathrm{g}$ of mitochondrial protein. The reaction was initiated by the addition of $20 \mu \mathrm{l}$ of $10 \mathrm{mM}$ oxaloacetate (final concentration $0.2 \mathrm{mM}$ ) (Folbergrová et al., 2007, 2016).

Activity of complex I was expressed as $\mathrm{nmol} / \mathrm{min} / \mathrm{mg}$ protein. To correct for the potential variations in mitochondrial contents in the samples, activities can also be expressed as a ratio to citrate synthase.

\section{Protein Determination}

Mitochondrial protein concentration was estimated by Bradford's method, with bovine serum albumin as a standard.

\section{Statistics}

Statistical analysis was performed in SigmaPlot 13 software (Systat Software Inc., United States). The data were evaluated by one-way ANOVA with Newmann-Keul's post hoc test or by $t$-test where appropriate. The level of statistical significance was set to $5 \%$.

\section{RESULTS}

\section{The Behavioral Pattern of Seizures}

All four convulsants induced SE that was characterized by generalized clonic-tonic seizures in DL-HCA and 4-AP models and by generalized clonic seizures in Li-Pilo and KA model, in the latter case accompanied by mild tonic extensions. Detailed 

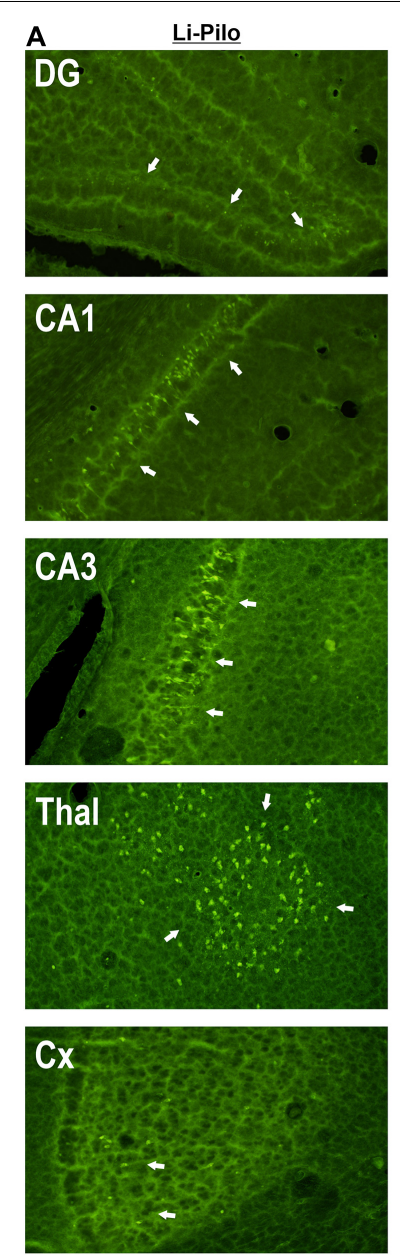
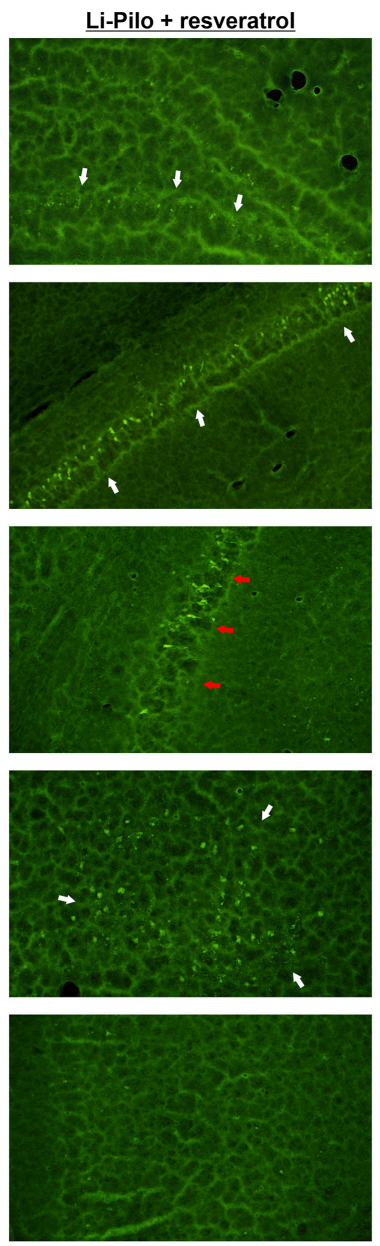

B

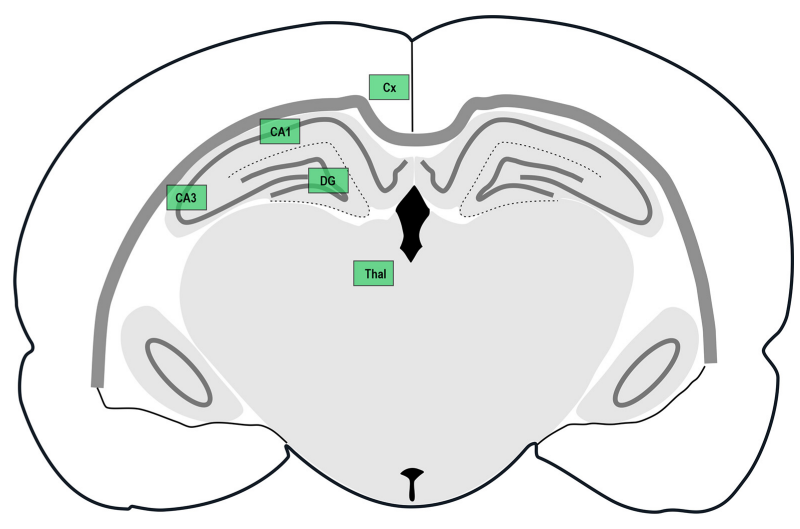

C

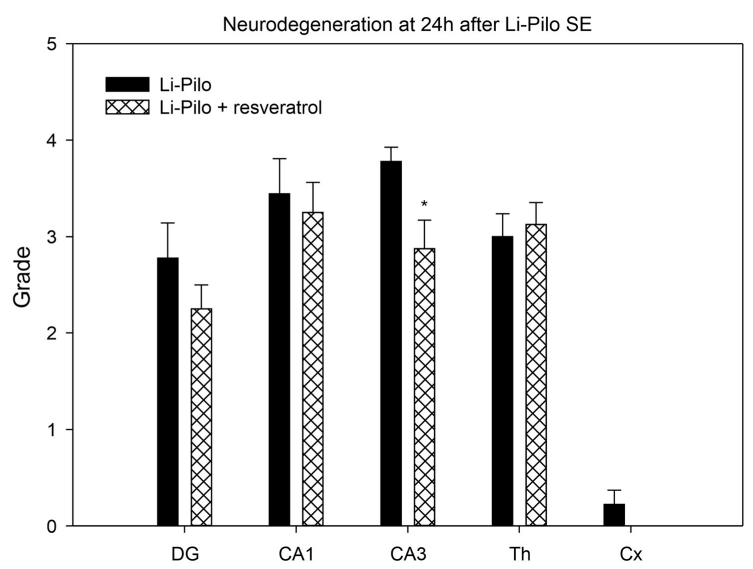

FIGURE 4 | Effect of resveratrol on brain damage. (A) Fluoro-Jade B staining revealed neurodegeneration in the explored regions (white arrows). Resveratrol treatment provided neuroprotective effect in CA3 region (red arrows). (B) Schema of investigated regions. (C) Brain damage evaluated by semiquantitative grading of FJB positive cells (description of score employed is given in the section "Materials and Methods"). * $P<0.05$ as compared with Li-Pilo alone.

description is given in our previous studies (Folbergrová et al., 2000, 2016).

\section{Effect of Resveratrol on Behavioral Pattern and on Electrographic Activity in Li-Pilo SE}

In all four models studied, latency to the first behavioral seizure and character of SE were not influenced by resveratrol. Effect of resveratrol on electrographic pattern has been analyzed in Li-Pilo model. Latency to the first electrographic seizure and severity of $\mathrm{SE}$, as assessed by number of spikes during first $90 \mathrm{~min}$, were not influenced by resveratrol treatment (Figure 2).

\section{Generation of Superoxide Anion During Seizures}

As can be seen in Figures 3A,B, fluorescent signal of the oxidized products of $\mathrm{Het}$ (reflecting $\mathrm{O}_{2}{ }^{-}$production) significantly increased in all the studied brain structures, namely CA1, CA3, and DG of hippocampus, cerebral cortex and thalamus of immature rats after SE lasting $60 \mathrm{~min}$ in all four models, with the exception of DG in DL-HCA model.

\section{Effect of Resveratrol on $\mathrm{O}_{2} \cdot{ }^{-}$Production}

Figure 3B (cross-hatched columns) demonstrates that RSV provided a complete protection in Li-Pilo, 4-AP and KA models and significantly reduced the fluorescence signal during SE induced by DL-HCA (see also Figure 3A, lower row of images).

\section{Effect of Resveratrol on Brain Damage}

Status epilepticus induced by Li-Pilocarpine resulted in a perceptible neuronal damage as revealed by Fluoro-Jade B staining at $24 \mathrm{~h}$ after SE. Structures of hippocampal formation, namely CA1 and CA3, as well as dentate gyrus (DG) and mediodorsal thalamic nuclei have been bilaterally affected and various number of Fluoro-Jade B positive cells has been identified under both control (Li-Pilo only) and resveratrol treated conditions. Sensorimotor cortex, however, revealed only a minimal or none neuronal damage. Semiquantitative evaluation 
of neuronal damage in selected regions of interest revealed partial but still significant neuroprotection in CA3 field of hippocampus (3.8 \pm 0.15 vs. $2.9 \pm 0.3, P=0.023)$ while other evaluated regions did not show signs of neuroprotection (Figures 4A,C).

\section{Effect of Resveratrol on Inhibition of Complex I Activity}

Our previous studies have demonstrated that SE induced in immature rats by DL-HCA (Folbergrová et al., 2007) or by 4AP, Li-Pilo, and KA (Folbergrová et al., 2016) leads to a marked deficiency of complex I activity, corresponding to more than 50\%. As evident in Figure 5, the inhibition of complex I activity was in all four models studied significantly attenuated by the treatment with RSV. Although significant, the protection was only partial since the activities of complex I after treatment with RSV remained significantly lower as compared with the appropriate controls. The same decrease of complex I activity and the same extent of protection provided by resveratrol was evident when the activity was expressed both as the specific activity (Figure 5) and as a ratio to citrate synthase (data not shown).

\section{DISCUSSION}

The important finding of the present study is the proof that resveratrol, naturally occurring polyphenolic compound, was able significantly reduce mitochondrial dysfunction associated with the acute phase of SE in immature rats.

The crucial question arises whether the protective effect of RSV could not be due to an anticonvulsant effect. However, behavioral pattern of seizures (severity, frequencies, duration) observed in all the studied models did not differ between groups

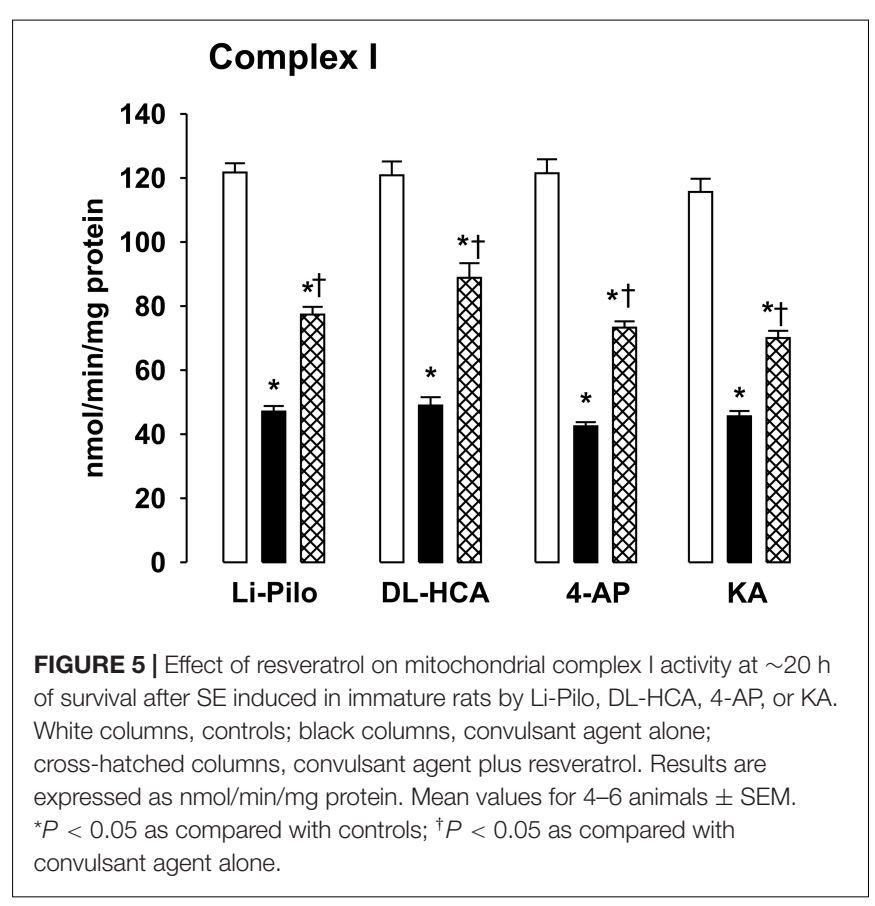

with convulsant agent and resveratrol and those with convulsant compound alone. In addition, lack of an anticonvulsant property of RSV was confirmed in Li-Pilo model by EEG recordings. Our findings are thus in agreement with recent reports of Tomaciello et al. (2016). These authors employing three different seizure models in adult mice did not observe an obvious anticonvulsant effect of RSV, only a trend toward a delay in seizure latency. Thus, our findings are compatible with the statement that the protective effect of RSV is most likely due to its antioxidant properties.

Neuroprotective effect of RSV has been observed in various models of neurological disorders in adult animals (Gupta et al., 2002; Wu et al., 2009; Kroon et al., 2010; Sakata et al., 2010; Shetty, 2011; Wang et al., 2013; Mishra et al., 2015; Castro et al., 2017). Its employment has some advantages, since RSV enters the brain after a peripheral administration and it does not seem to have adverse effects (Shetty, 2011). It seems likely that substances interacting with multiple targets can achieve a better effect than single target therapies. Thus, RSV besides the direct antioxidant effect (Holthoff et al., 2010; Folbergrová et al., 2015) has multiple cellular effects, interfering with several signaling pathways (Kroon et al., 2010; Sahebkar, 2010; Shetty, 2011; Castro et al., 2017). Recently, it has been reported that RSV is able to activate Nrf2 (nuclear factor erythroid 2-related factor 2) which is an essential transcription factor regulating the expression of numerous endogenous antioxidant and anti-inflammatory genes and plays a crucial role in cellular defense against oxidative stress (Kesherwani et al., 2013; Narayanan et al., 2015). Importantly, our recent findings indicate that neuroprotective effect comparable to that observed with RSV can be detected in immature rats during Li-pilocarpine SE after treatment with sulforaphane, established activator of Nrf2 (manuscript in preparation). Furthermore, an increased expression (Mazzuferi et al., 2013) or activation of Nrf2 (Wang et al., 2014) have been reported recently to provide a marked protection in experimental epilepsy models in adults. Nevertheless, the precise mechanism of RSV action in our study remains to be clarified by future studies.

Whatever the mechanism, RSV prevented completely the generation of $\mathrm{O}_{2} \cdot-$ associated with the acute phase of SE. It should be mentioned that the Het assay used for the evaluation of $\mathrm{O}_{2} \cdot{ }^{-}$formation has several limitations as discussed recently (Zielonka and Kalyanaraman, 2010; Kalyanaraman et al., 2014, 2017). These mainly concern the difficulty to distinguish microscopically the fluorescent red signal belonging to 2hydroxyethidium (a specific product of Het reaction with $\mathrm{O}_{2}$ - ) and ethidium (a product of Het reaction with other ROS and/or oxidants). Importantly, our recent findings demonstrating complete prevention of the increased fluorescent signal after the treatment animals with SOD mimetic MnTMPYP, support the involvement of superoxide anion (Folbergrová et al., 2012, 2016).

Mitochondrial dysfunction, especially a deficiency of complex I activity has been demonstrated in humans with temporal lobe epilepsy (Kunz et al., 2000; Lee et al., 2008) and in several experimental models of epilepsy in adult (Kudin et al., 2002; Chuang et al., 2004; Waldbaum and Patel, 2010; Shin et al., 2011) as well as immature animals during SE (Folbergrová et al., 2007, 2010, 2016). In agreement with our previous studies, more than $50 \%$ decrease of complex I activity was observed 
in all four models of SE induced in immature rats (Figure 5). The question arises what may be the underlying mechanism. We showed that in DL-HCA model, the decrease of complex I activity was not associated with changes in the size of the assembled complex I or with changes in mitochondrial content of complex I (Folbergrová et al., 2010). We have thus proposed that inactivation, namely oxidative modification of complex I, may be responsible for the deficiency of complex I activity. This assumption is supported by an extreme sensitivity of this enzyme to both oxidative and nitrosative stress (Folbergrová et al., 2010 and references therein). Furthermore, the increased ROS production detected in all four models can create conditions favoring oxidative modifications of sensitive targets. Several posttranslational oxidative modifications of complex I can occur, such as carboxylation, nitration of tyrosine (and/or tryptophane) residues, S-nitrosation of some of its protein thiols etc. (Murray et al., 2003; Brown and Borutaite, 2004; Folbergrová et al., 2010; Ryan et al., 2012, 2014). Indeed, the oxidative modification (nitration or carboxylation) of only a few subunits from the total 46 was reported to result in a pronounced inhibition of complex I activity (Murray et al., 2003; Ryan et al., 2012). Potential role of other factors beside oxidative inactivation cannot, however, be excluded. Nevertheless, the involvement of oxidative modification is supported by our recent findings demonstrating that deficiency of complex I activity could be significantly attenuated by SOD mimics Tempol (Folbergrová et al., 2007) or MnTMPYP (Folbergrová et al., 2010, 2016), by a selective peroxynitrite scavenger and decomposition catalyst FeTPPS (Folbergrová et al., 2007, 2010, 2016) and, as the present findings clearly indicate, by resveratrol.

It should be kept in mind that complex I besides being a target for ROS and RNS is also the important source of their production, especially when partially inhibited (Sipos et al., 2003; Kudin et al., 2004; Kussmaul and Hirst, 2006; Fato et al., 2008; Parihar et al., 2008). It can thus be assumed that the enhanced production of ROS and/or RNS as a consequence of complex I inhibition, may lead to a potential impairment of sufficient energy production, contribute to neuronal injury and/or epileptogenesis (Perier et al., 2005; Marella et al., 2007; Castro et al., 2017).

\section{CONCLUSION}

The present study clearly demonstrates that treatment with resveratrol significantly attenuates early mitochondrial dysfunction (evident as a marked preservation of complex I activity) during the acute phase of status epilepticus in immature

\section{REFERENCES}

Batinic-Haberle, I., Reboucas, J. S., and Spasojevic, I. (2010). Superoxide dismutase mimics: chemistry, pharmacology, and therapeutic potential. Antioxid Redox Signal 13, 877-918. doi: 10.1089/ars.2009.2876

Bindokas, V. P., Jordan, J., Lee, C. C., and Miller, R. J. (1996). Superoxide production in rat hippocampal neurons: selective imaging with hydroethidine. J. Neurosci. 16, 1324-1336. doi: 10.1523/jneurosci.16-04-01324. 1996 rats. The protective effect of resveratrol was evident in all four models, induced in immature rats with substances of a different mechanism of action and, can thus represent a general phenomenon associated with SE in immature brain. Since resveratrol does not influence seizure activity itself, the mechanism of protective action is most likely due to its antioxidative properties (as documented by diminished $\mathrm{O}_{2}{ }^{-}$ production). The findings have a clinical relevance suggesting that clinically available substances with antioxidant properties might provide a high benefit as an add-on therapy during the acute phase of status epilepticus interacting with mechanisms involved in development of epilepsy.

\section{DATA AVAILABILITY STATEMENT}

The raw data supporting the conclusions of this article will be made available by the authors, without undue reservation.

\section{ETHICS STATEMENT}

The animal study was reviewed and approved by the Ethical and Use committee of the Institute of Physiology CAS, Prague.

\section{AUTHOR CONTRIBUTIONS}

JF: conceptualization and writing-original draft. JF and JO: formal analysis, supervision, visualization, and writing-review and editing. JO: funding acquisition and project administration. JF, PJ, and JO: investigation and methodology. All authors contributed to the article and approved the submitted version.

\section{FUNDING}

This study was supported by the grants \#15-08565S and \#1807908S from the Czech Science Foundation and with institutional support by Czech Academy of Sciences through "QUALITASWellbeing in health and disease" and RVO: 67985823.

\section{ACKNOWLEDGMENTS}

We express our thanks to Mrs. E. Lažková for her excellent technical assistance.

Brown, G. C., and Borutaite, V. (2004). Inhibition of mitochondrial respiratory complex I by nitric oxide, peroxynitrite and S-nitrosothiols. Biochim. Biophys. Acta 1658, 44-49. doi: 10.1016/j.bbabio.2004.03.016

Castro, O. W., Upadhya, D., Kodali, M., and Shetty, A. K. (2017). Resveratrol for easing status epilepticus induced brain injury, inflammation, epileptogenesis, and cognitive and memory dysfunction-are we there yet? Front. Neurol. 8:603. doi: 10.3389/fneur.2017.00603

Chuang, Y. C., Chang, A. Y., Lin, J. W., Hsu, S. P., and Chan, S. H. (2004). Mitochondrial dysfunction and ultrastructural damage in the hippocampus 
during kainic acid-induced status epilepticus in the rat. Epilepsia 45, 1202-1209. doi: 10.1111/j.0013-9580.2004.18204.x

Dobbing, J. (1970). “Undernutrition and the developing brain," in Developmental Neurobiology, eds W. A. Himwich and C. C. Thomas (Hoboken, NJ: WileyBlackwell), 241-261.

Fato, R., Bergamini, C., Leoni, S., Strocchi, P., and Lenaz, G. (2008). Generation of reactive oxygen species by mitochondrial complex I: implications in neurodegeneration. Neurochem. Res. 33, 2487-2501. doi: 10.1007/s11064-0089747-0

Folbergrová, J. (2013). Oxidative stress in immature brain following experimentally-induced seizures. Physiol. Res. 62(Suppl. 1), S39-S48.

Folbergrová, J. (2016). "Free radicals, oxidative stress, and epilepsy," in Reactive Oxygen Species in Biology and Human Health, ed. A. Shamim (Boca Raton: CRC Press), 147-153.

Folbergrová, J., Haugvicová, R., and Mareš, P. (2000). Behavioral and metabolic changes in immature rats during seizures induced by homocysteic acid: the protective effect of NMDA and non-NMDA receptor antagonists. Exp. Neurol. 161, 336-345. doi: 10.1006/exnr.1999.7264

Folbergrová, J., Ješina, P., Drahota, Z., Lisl, V., Haugvicová, R., Vojtíšková, A., et al. (2007). Mitochondrial complex I inhibition in cerebral cortex of immature rats following homocysteic acid-induced seizures. Exp. Neurol. 204, 597-609. doi: 10.1016/j.expneurol.2006.12.010

Folbergrová, J., Ješina, P., Haugvicová, R., Lis $\iota$, V., and Houštik, J. (2010). Sustained deficiency of mitochondrial complex I activity during long periods of survival after seizures induced in immature rats by homocysteic acid. Neurochem. Int. 56, 394-403. doi: 10.1016/j.neuint.2009.11.011

Folbergrová, J., Ješina, P., Kubová, H., Druga, R., and Otáhal, J. (2016). Status epilepticus in immature rats is associated with oxidative stress and mitochondrial dysfunction. Front. Cell. Neurosci. 10:136. doi: 10.3339/fncel. 2016.00136

Folbergrová, J., Ješina, P., Kubová, H., and Otáhal, J. (2015). Resveratrol attenuates oxidative stress associated with status epilepticus in immature rats. Epilepsia 56, 118-118.

Folbergrová, J., Ješina, P., Kubová, H., and Otáhal, J. (2018). Effect of resveratrol on oxidative stress and mitochondrial dysfunction in immature brain during epileptogenesis. Mol. Neurobiol. 55, 7512-7522. doi: 10.1007/s12035-0180924-0

Folbergrová, J., and Kunz, W. S. (2012). Mitochondrial dysfunction in epilepsy. Mitochondrion 12, 35-40. doi: 10.1016/j.mito.2011.04.004

Folbergrová, J., Otáhal, J., and Druga, R. (2011). Effect of tempol on brain superoxide anion production and neuronal injury associated with seizures in immature rats. Epilepsia 52, 51-51.

Folbergrová, J., Otáhal, J., and Druga, R. (2012). Brain superoxide anion formation in immature rats during seizures: protection by selected compounds. Exp. Neurol. 233, 421-429. doi: 10.1016/j.expneurol.2011.11.009

Gupta, Y. K., Briyal, S., and Chaudhary, G. (2002). Protective effect of transresveratrol against kainic acid-induced seizures and oxidative stress in rats. Pharmacol. Biochem. Behav. 71, 245-249. doi: 10.1016/s0091-3057(01)00663-3

Holthoff, J. H., Woodling, K. A., Doerge, D. R., Burns, S. T., Hinson, J. A., and Mayeux, P. R. (2010). Resveratrol, a dietary polyphenolic phytoalexin, is a functional scavenger of peroxynitrite. Biochem. Pharmacol. 80, 1260-1265. doi: 10.1016/j.bcp.2010.06.027

Kalyanaraman, B., Dranka, B. P., Hardy, M., Michalski, R., and Zielonka, J. (2014). HPLC-based monitoring of products formed from hydroethidinebased fluorogenic probes-the ultimate approach for intra- and extracellular superoxide detection. Biochim. Biophys. Acta 1840, 739-744. doi: 10.1016/j. bbagen.2013.05.008

Kalyanaraman, B., Hardy, M., Podsiadly, R., Cheng, G., and Zielonka, J. (2017). Recent developments in detection of superoxide radical anion and hydrogen peroxide: opportunities, challenges, and implications in redox signaling. Arch. Biochem. Biophys. 617, 38-47. doi: 10.1016/j.abb.2016.08.021

Kesherwani, V., Atif, F., Yousuf, S., and Agrawal, S. K. (2013). Resveratrol protects spinal cord dorsal column from hypoxic injury by activating Nrf-2. Neuroscience 241, 80-88. doi: 10.1016/j.neuroscience.2013.03.015

Kroon, P. A., Iyer, A., Chunduri, P., Chan, V., and Brown, L. (2010). The cardiovascular nutrapharmacology of resveratrol: pharmacokinetics, molecular mechanisms and therapeutic potential. Curr. Med. Chem. 17, 2442-2455. doi: $10.2174 / 092986710791556032$
Kudin, A. P., Bimpong-Buta, N. Y., Vielhaber, S., Elger, C. E., and Kunz, W. S. (2004). Characterization of superoxide-producing sites in isolated brain mitochondria. J. Biol. Chem. 279, 4127-4135. doi: 10.1074/jbc.M310341200

Kudin, A. P., Kudina, T. A., Seyfried, J., Vielhaber, S., Beck, H., Elger, C. E., et al. (2002). Seizure-dependent modulation of mitochondrial oxidative phosphorylation in rat hippocampus. Eur. J. Neurosci. 15, 1105-1114. doi: 10.1046/j.1460-9568.2002.01947.x

Kunz, W. S., Kudin, A. P., Vielhaber, S., Blumcke, I., Zuschratter, W., Schramm, J., et al. (2000). Mitochondrial complex I deficiency in the epileptic focus of patients with temporal lobe epilepsy. Ann. Neurol. 48, 766-773.

Kussmaul, L., and Hirst, J. (2006). The mechanism of superoxide production by NADH:ubiquinone oxidoreductase (complex I) from bovine heart mitochondria. Proc. Natl. Acad. Sci. U.S.A. 103, 7607-7612. doi: 10.1073/pnas. 0510977103

Lee, Y. M., Kang, H. C., Lee, J. S., Kim, S. H., Kim, E. Y., Lee, S. K., et al. (2008). Mitochondrial respiratory chain defects: underlying etiology in various epileptic conditions. Epilepsia 49, 685-690. doi: 10.1111/j.1528-1167.2007.01522.x

Liang, L. P., Ho, Y. S., and Patel, M. (2000). Mitochondrial superoxide production in kainate-induced hippocampal damage. Neuroscience 101, 563-570.

Liang, L. P., Waldbaum, S., Rowley, S., Huang, T. T., Day, B. J., and Patel, M. (2012). Mitochondrial oxidative stress and epilepsy in SOD2 deficient mice: attenuation by a lipophilic metalloporphyrin. Neurobiol. Dis. 45, 1068-1076. doi: 10.1016/j.nbd.2011.12.025

Linseman, D. A. (2009). Targeting oxidative stress for neuroprotection. Antioxid Redox Signal 11, 421-424. doi: 10.1089/ARS.2008.2236

Marella, M., Seo, B. B., Matsuno-Yagi, A., and Yagi, T. (2007). Mechanism of cell death caused by complex I defects in a rat dopaminergic cell line. J. Biol. Chem. 282, 24146-24156. doi: 10.1074/jbc.M701819200

Mazzuferi, M., Kumar, G., Van Eyll, J., Danis, B., Foerch, P., and Kaminski, R. M. (2013). Nrf2 defense pathway: experimental evidence for its protective role in epilepsy. Ann. Neurol. 74, 560-568. doi: 10.1002/ana.23940

Mishra, V., Shuai, B., Kodali, M., Shetty, G. A., Hattiangady, B., Rao, X., et al. (2015). Resveratrol treatment after status epilepticus restrains neurodegeneration and abnormal neurogenesis with suppression of oxidative stress and inflammation. Sci. Rep. 5:17807. doi: 10.1038/srep17807

Murray, J., Taylor, S. W., Zhang, B., Ghosh, S. S., and Capaldi, R. A. (2003). Oxidative damage to mitochondrial complex I due to peroxynitrite: identification of reactive tyrosines by mass spectrometry. J. Biol. Chem. 278, 37223-37230. doi: 10.1074/jbc.M305694200

Narayanan, S. V., Dave, K. R., Saul, I., and Perez-Pinzon, M. A. (2015). Resveratrol preconditioning protects against cerebral ischemic injury via nuclear erythroid 2-Related factor 2. Stroke 46, 1626-1632. doi: 10.1161/STROKEAHA.115. 008921

Parihar, M. S., Parihar, A., Villamena, F. A., Vaccaro, P. S., and Ghafourifar, P. (2008). Inactivation of mitochondrial respiratory chain complex I leads mitochondrial nitric oxide synthase to become pro-oxidative. Biochem. Biophys. Res. Commun. 367, 761-767. doi: 10.1016/j.bbrc.2008.01.015

Patel, M. (2004). Mitochondrial dysfunction and oxidative stress: cause and consequence of epileptic seizures. Free Radic. Biol. Med. 37, 1951-1962. doi: 10.1016/j.freeradbiomed.2004.08.021

Patel, M., and Day, B. J. (1999). Metalloporphyrin class of therapeutic catalytic antioxidants. Trends Pharmacol. Sci. 20, 359-364. doi: 10.1016/s0165-6147(99) 01336-x

Pauletti, A., Terrone, G., Shekh-Ahmad, T., Salamone, A., Ravizza, T., Rizzi, M., et al. (2017). Targeting oxidative stress improves disease outcomes in a rat model of acquired epilepsy. Brain 140, 1885-1899. doi: 10.1093/brain/awx117

Paxinos, G., and Watson, C. (1998). The Rat Brain in Stereotaxic Coordinates. San Diego: Academic Press.

Perier, C., Tieu, K., Guegan, C., Caspersen, C., Jackson-Lewis, V., Carelli, V., et al. (2005). Complex I deficiency primes Bax-dependent neuronal apoptosis through mitochondrial oxidative damage. Proc. Natl. Acad. Sci. U.S.A. 102, 19126-19131. doi: 10.1073/pnas.0508215102

Reboucas, J. S., Spasojevic, I., and Batinic-Haberle, I. (2008). Quality of potent $\mathrm{Mn}$ porphyrin-based SOD mimics and peroxynitrite scavengers for pre-clinical mechanistic/therapeutic purposes. J. Pharm. Biomed. Anal. 48, 1046-1049. doi: 10.1016/j.jpba.2008.08.005

Rong, Y., Doctrow, S. R., Tocco, G., and Baudry, M. (1999). EUK-134, a synthetic superoxide dismutase and catalase mimetic, prevents oxidative stress and 
attenuates kainate-induced neuropathology. Proc. Natl. Acad. Sci. U.S.A. 96, 9897-9902. doi: 10.1073/pnas.96.17.9897

Rowley, S., and Patel, M. (2013). Mitochondrial involvement and oxidative stress in temporal lobe epilepsy. Free Radic. Biol. Med. 62, 121-131. doi: 10.1016/j. freeradbiomed.2013.02.002

Ryan, K., Backos, D. S., Reigan, P., and Patel, M. (2012). Post-translational oxidative modification and inactivation of mitochondrial complex I in epileptogenesis. J. Neurosci. 32, 11250-11258. doi: 10.1523/JNEUROSCI.090712.2012

Ryan, K., Liang, L. P., Rivard, C., and Patel, M. (2014). Temporal and spatial increase of reactive nitrogen species in the kainate model of temporal lobe epilepsy. Neurobiol. Dis. 64, 8-15. doi: 10.1016/j.nbd.2013.12.006

Sahebkar, A. (2010). Neuroprotective effects of resveratrol: potential mechanisms. Neurochem. Int. 57, 621-622. doi: 10.1016/j.neuint.2010.06.014

Sakata, Y., Zhuang, H., Kwansa, H., Koehler, R. C., and Dore, S. (2010). Resveratrol protects against experimental stroke: putative neuroprotective role of heme oxygenase 1. Exp. Neurol. 224, 325-329. doi: 10.1016/j.expneurol.2010.03.032

Sheng, H., Chaparro, R. E., Sasaki, T., Izutsu, M., Pearlstein, R. D., Tovmasyan, A., et al. (2014). Metalloporphyrins as therapeutic catalytic oxidoreductants in central nervous system disorders. Antioxid Redox Signal 20, 2437-2464. doi: 10.1089 /ars.2013.5413

Shetty, A. K. (2011). Promise of resveratrol for easing status epilepticus and epilepsy. Pharmacol. Ther. 131, 269-286. doi: 10.1016/j.pharmthera.2011.04. 008

Shin, E. J., Jeong, J. H., Chung, Y. H., Kim, W. K., Ko, K. H., Bach, J. H., et al. (2011). Role of oxidative stress in epileptic seizures. Neurochem. Int. 59, 122-137. doi: 10.1016/j.neuint.2011.03.025

Sipos, I., Tretter, L., and Adam-Vizi, V. (2003). Quantitative relationship between inhibition of respiratory complexes and formation of reactive oxygen species in isolated nerve terminals. J. Neurochem. 84, 112-118.

Tomaciello, F., Leclercq, K., and Kaminski, R. M. (2016). Resveratrol lacks protective activity against acute seizures in mouse models. Neurosci. Lett. 632, 199-203. doi: 10.1016/j.neulet.2016.09.002
Waldbaum, S., and Patel, M. (2010). Mitochondria, oxidative stress, and temporal lobe epilepsy. Epilepsy Res. 88, 23-45. doi: 10.1016/j.eplepsyres.2009.09.020

Wang, S. J., Bo, Q. Y., Zhao, X. H., Yang, X., Chi, Z. F., and Liu, X. W. (2013). Resveratrol pre-treatment reduces early inflammatory responses induced by status epilepticus via mTOR signaling. Brain Res. 1492, 122-129. doi: 10.1016/j. brainres.2012.11.027

Wang, W., Wu, Y., Zhang, G., Fang, H., Wang, H., Zang, H., et al. (2014). Activation of Nrf2-ARE signal pathway protects the brain from damage induced by epileptic seizure. Brain Res. 1544, 54-61. doi: 10.1016/j.brainres.2013. 12.004

Williams, S., Hamil, N., Abramov, A. Y., Walker, M. C., and Kovac, S. (2015). Status epilepticus results in persistent overproduction of reactive oxygen species, inhibition of which is neuroprotective. Neuroscience 303, 160-165. doi: 10.1016/ j.neuroscience.2015.07.005

Wu, Z., Xu, Q., Zhang, L., Kong, D., Ma, R., and Wang, L. (2009). Protective effect of resveratrol against kainate-induced temporal lobe epilepsy in rats. Neurochem. Res. 34, 1393-1400. doi: 10.1007/s11064-0099920-0

Zielonka, J., and Kalyanaraman, B. (2010). Hydroethidine- and MitoSOX-derived red fluorescence is not a reliable indicator of intracellular superoxide formation: another inconvenient truth. Free Radic. Biol. Med. 48, 983-1001. doi: 10.1016/j. freeradbiomed.2010.01.028

Conflict of Interest: The authors declare that the research was conducted in the absence of any commercial or financial relationships that could be construed as a potential conflict of interest.

Copyright (C) 2021 Folbergrová, Ješina and Otáhal. This is an open-access article distributed under the terms of the Creative Commons Attribution License (CC BY). The use, distribution or reproduction in other forums is permitted, provided the original author(s) and the copyright owner(s) are credited and that the original publication in this journal is cited, in accordance with accepted academic practice. No use, distribution or reproduction is permitted which does not comply with these terms. 